Directions and equidistribution in homology for periodic orbits

Collier, David and Sharp, Richard

2006

MIMS EPrint: 2006.49

Manchester Institute for Mathematical Sciences

School of Mathematics

The University of Manchester

\footnotetext{
Reports available from: http://eprints.maths.manchester.ac.uk/

And by contacting: The MIMS Secretary

School of Mathematics

The University of Manchester

Manchester, M13 9PL, UK
} 


\title{
DIRECTIONS AND EQUIDISTRIBUTION IN HOMOLOGY FOR PERIODIC ORBITS
}

\author{
David Collier AND Richard Sharp \\ University of Manchester
}

\begin{abstract}
We discuss the asymptotic distribution of the directions in homology of periodic orbits of Anosov flows. We obtaining a limiting measure which is either a Dirac measure on a single point or is fully supported. In the latter case, we relate the result to a more general equidistribution problem.
\end{abstract}

\section{IntroduCtion AND Results}

Let $M$ be a compact smooth Riemannian manifold and let $\phi_{t}: M \rightarrow M$ be a transitive Anosov flow. Such a flow has a countable infinity of (prime) periodic orbits $\gamma$ with length $l(\gamma)$. Write $\pi(T)=\#\{\gamma: l(\gamma) \leq T\}$; then a classical result of Margulis [8],[9] states that $\pi(T) \sim e^{h(\phi) T} / h(\phi) T$, as $T \rightarrow+\infty$, where $h(\phi)>0$ is the topological entropy of $\phi$.

A more delicate problem is to understand the distribution of periodic orbits with respect to the homology group $H_{1}(M, \mathbb{Z})$, which we suppose to be infinite. To simplify notation, we shall work modulo torsion and, in addition, suppose that a basis has been fixed so that we may write $H_{1}(M, \mathbb{Z})=\mathbb{Z}^{k}$ for some $k \geq 1$. Then, for $\alpha \in H_{1}(M, \mathbb{Z})$, we may try to study $\pi(T, \alpha)=\#\{\gamma: l(\gamma) \leq T,[\gamma]=\alpha\}$, where $[\gamma]$ denotes the homology class of $\gamma$. There is a basic dichotomy which may be characterized in terms of the winding cycle (or asymptotic cycle) $\Phi_{0} \in H_{1}(M, \mathbb{R})$ associated to the measure of maximal entropy of $\phi$ [14]. If $\Phi_{0}=0$ then, for some constant $C>0$ (independent of $\alpha$ ),

$$
\pi(T, \alpha) \sim C \frac{e^{h(\phi) T}}{T^{k / 2+1}}, \text { as } T \rightarrow+\infty
$$

while if $\Phi_{0} \neq 0$ then $\pi(T, \alpha) / \pi(T)$ tends to zero exponentially fast, as $T \rightarrow+\infty$.

In this paper we shall study the distribution of directions of homology classes. If $[\gamma] \neq 0$ then we define $\theta(\gamma)$ to be the projection of $[\gamma]$ to $\left(H_{1}(M, \mathbb{R}) \backslash\{0\}\right) / \mathbb{R}^{+}$, the set of (non-zero) directions in $H_{1}(M, \mathbb{R})$. It is convenient to parametrize these directions by $S^{k-1}=\{x \in$ $\left.\mathbb{R}^{k}:\|x\|_{2}=1\right\}$, where $\|\cdot\|_{2}$ is the usual 2-norm. (Recall that there is a fixed isomorphism between $H_{1}(M, \mathbb{R})$ and $\mathbb{R}^{k}$.)

In [3], Fried introduced, for an arbitrary flow on a compact manifold, the set $\mathcal{D}_{\phi} \subset S^{k-1}$ of homology directions for the flow. (Actually, Fried allows the zero direction.) Roughly speaking, these correspond to the normalized homology classes of long almost closed orbits. 
Fried showed that $\phi$ has a global cross section if and only if $\mathcal{D}_{\phi}$ is contained in an open hemisphere in $S^{k-1}$. In the Anosov case, we have the alternative characterization

$$
\mathcal{D}_{\phi}=\overline{\{\theta(\gamma):[\gamma] \neq 0\}},
$$

the closure of the set of directions of periodic orbits, and it follows from results in [15] that either $\mathcal{D}_{\phi}=S^{k-1}$ or $\mathcal{D}_{\phi}$ is contained in an closed hemisphere in $S^{k-1}$. In particular, if $\Phi_{0}=0$ then $\mathcal{D}_{\phi}=S^{k-1}$ but the converse is not true.

We wish to understand the asymptotic distribution of the directions $\theta(\gamma)$ within $\mathcal{D}_{\phi}$. Let $\delta_{\theta(\gamma)}$ denote the Dirac measure at $\theta(\gamma)$; we shall consider the behaviour, as $T \rightarrow+\infty$, of the measures

$$
\nu_{\phi}^{T}=\frac{1}{\pi(T)} \sum_{\substack{l(\gamma) \leq T \\[\gamma] \neq 0}} \delta_{\theta(\gamma)} .
$$

(One knows that $\#\{\gamma: l(\gamma) \leq T,[\gamma] \neq 0\} \sim \pi(T)$, as $T \rightarrow+\infty$, so the normalization in (0.2) is appropriate.)

Theorem 1. Let $\phi_{t}: M \rightarrow M$ be a transitive Anosov flow. The measures $\nu_{\phi}^{T}$ have a weak* limit $\nu_{\phi}$, as $T \rightarrow+\infty$.

(i) If $\Phi_{0}=0$ then $\nu_{\phi}$ has full support on $S^{k-1}$. More precisely, there is a norm $\|\cdot\|$ on $H_{1}(M, \mathbb{R})$ (obtained from an inner product) such that, for a Borel set $D \subset S^{k-1}$ whose boundary has zero measure,

$$
\nu_{\phi}(D)=\frac{\operatorname{Vol}_{k}\left(S(D) \cap B_{\|\cdot\|}\right)}{\operatorname{Vol}_{k}\left(B_{\|\cdot\|}\right)},
$$

where $S(D)=\{\lambda \omega: \omega \in D, \lambda>0\}$ is the sector based on $D$ and $B_{\|\cdot\|}$ is the unit ball for $\|\cdot\|$.

(ii) If $\Phi_{0} \neq 0$ then $\nu_{\phi}$ is equal to the the Dirac measure at $\Phi_{0} /\left\|\Phi_{0}\right\|_{2}$.

The definition of the norm $\|\cdot\|$ is given in section 1 .

Remarks.

(i) An analogue to (0.1) holds (albeit with a smaller growth rate) provided that $\phi$ is homologically full, i.e., that the natural map from the set of periodic orbits to $H_{1}(M, \mathbb{Z})$ is a surjection [15]. This also has a characterization in terms of the vanishing of winding cycles associated to a rather natural class of measures. Under certain restricted circumstances, stronger results hold: asymptotic expansions for $\pi(T, \alpha)[1],[5],[13]$ and central and local limit theorems for $\pi(T, \alpha) / \pi(T)$ [2],[7],[16]. The norm mentioned in Theorem 1, explicitly identified in [5], appears in these results, a summary of which may be found in the second author's survey in [9].

(ii) It follows from [15] that $\phi$ is homologically full if and only if $\mathcal{D}_{\phi}=S^{k-1}$. Since there are homologically full flows for which $\Phi_{0} \neq 0$, Theorem 1 shows that, in particular, the support of $\nu_{\phi}$ is not necessarily equal to $\mathcal{D}_{\phi}$.

Theorem 1 may be rewritten as a statement about closed geodesics whose homology classes lie within a sector. Let $S_{\mathbb{Z}}(D)=S(D) \cap \mathbb{Z}^{k}$ be the set of homology classes lying in 
the sector based on $D$. For any $A \subset \mathbb{Z}^{k}$, let $\pi(T, A)=\#\{\gamma: l(\gamma) \leq T,[\gamma] \in A\}$. Then part (i) of Theorem 1 is equivalent to the statement that

$$
\lim _{T \rightarrow+\infty} \frac{\pi\left(T, S_{\mathbb{Z}}(D)\right)}{\pi(T)}=\nu_{\phi}(D) .
$$

Furthermore, we can reinterpret $\nu_{\phi}(D)$ as the density of $S_{\mathbb{Z}}(D)$ with respect to the norm $\|\cdot\|$, where the density $d_{\|\cdot\|}(A)$ of a set $A \subset \mathbb{Z}^{k}$ is defined by

$$
d_{\|\cdot\|}(A)=\lim _{r \rightarrow+\infty} \frac{\#\{\alpha \in A:\|\alpha\| \leq r\}}{\#\left\{\alpha \in \mathbb{Z}^{k}:\|\alpha\| \leq r\right\}}
$$

(whenever the limit exists). One can then view Theorem 1(i) as an equidistribution property of the periodic orbits: the proportion lying in $S_{\mathbb{Z}}(D)$ converges to the density of $S_{\mathbb{Z}}(D)$. In fact, we shall show that the analogue of (0.3) holds whenever the limit in (0.4) exists. Part (i) of Theorem 1 will then follow as a consequence.

Theorem 2. Let $\phi_{t}: M \rightarrow M$ be a transitive Anosov flow such that $\Phi_{0}=0$. If $A \subset \mathbb{Z}^{k}$ is a set for which the density $d_{\|\cdot\|}(A)$ exists then

$$
\lim _{T \rightarrow+\infty} \frac{\pi(T, A)}{\pi(T)}=d_{\|\cdot\|}(A)
$$

Remark. A prototypical example of a transitive Anosov flow with $\Phi_{0}$ is the geodesic flow on the unit tangent bundle $M=S N$ of a compact Riemannian manifold $N$ with negative sectional curvatures. Then, if $\operatorname{dim} N \geq 3, H_{1}(M, \mathbb{Z})=H_{1}(N, \mathbb{Z})$, while if $\operatorname{dim} N=2$, the two first homology groups agree up to torsion. In this setting, periodic orbits of the flow are in a natural one-to-one correspondence with closed geodesic on $N$ which preserves length and homology. Hence, under this hypothesis, Theorem 2 holds for closed geodesics. This result was recently obtained by Petridis and Risager in the case where $N$ is a compact hyperbolic surface [12]. A variation of their technique, taking as its starting point the analysis in [16], can be used in the case of surfaces with variable negative curvature and higher dimensional manifolds for which the metric is $1 / 4$-pinched.

We shall now outline the contents of the paper. In section 1, we discuss winding cycles associated to Anosov flows and their relationship to thermodynamic formalism, in particular defining the norm $\|\cdot\|$. In section 2 , we relate this theory to periodic orbits and state the results we need on their distribution and prove Theorem 1(ii). In section 3, we use these results to prove Theorem 2. The proof relies on convergence of certain Gaussian sums to a density; this follows from a technical lemma proved in section 4 .

Notational comment. Given functions $f(T)$ and $g(T)>0$, we shall write $f(T) \sim g(T)$ if $\lim _{T \rightarrow+\infty} f(T) / g(T)=1, f(T)=o(g(T))$ if $\lim _{T \rightarrow+\infty}|f(T)| / g(T)=0$, and $f(T)=$ $O(g(T))$ if there exists $C>0$ such that $|f(T)| \leq C g(T)$.

We are grateful to François Ledrappier, who asked the second author about the distribution of "homology directions" for periodic orbits. 


\section{Anosov Flows And a Norm on Homology}

Let $M$ be a smooth compact Riemannian manifold and let $\phi_{t}: M \rightarrow M$ be a $C^{1}$ flow. We say that $\phi$ is an Anosov flow if there is a continuous splitting $T M=E^{0} \oplus E^{s} \oplus E^{u}$, where $E^{0}$ is the line bundle to the flow and where, for constants $C>0$ and $\lambda>0$,

(1) $\left\|D \phi_{t}(v)\right\| \leq C e^{-\lambda t}\|v\|$, for all $v \in E^{s}$ and $t \geq 0$; and

(2) $\left\|D \phi_{-t}(v)\right\| \leq C e^{-\lambda t}\|v\|$, for all $v \in E^{u}$ and $t \geq 0$.

If, in addition, $\phi$ has a dense orbit then we say that $\phi$ is transitive. A prototypical example of a (transitive) Anosov flow is the geodesic flow on the unit-tangent bundle $S N$ over a compact Riemannian manifold $N$ with negative sectional curvatures.

We shall suppose that $H_{1}(M, \mathbb{Z})$ is infinite, so that $H_{1}(M, \mathbb{R}) \cong \mathbb{R}^{k}$, for some $k \geq 1$. Let $\mathcal{M}_{\phi}$ denote the set of all $\phi$-invariant probability measures on $M$. The homological distribution of the periodic orbits described in the next section is intimately related to a family of real homology classes associated to elements of $\mathcal{M}_{\phi}$. For $\mu \in \mathcal{M}_{\phi}$, we define the winding cycle (or asymptotic cycle) $\Phi_{\mu} \in H_{1}(M, \mathbb{R})$ as a functional on the (de Rham) cohomology $H^{1}(M, \mathbb{R})$ given by

$$
\Phi_{\mu}([\omega])=\int \omega(\mathcal{X}) d \mu,
$$

where $\omega$ is a closed 1 -form in the cohomology class $[\omega]$ and $\mathcal{X}$ is the vector field tangent to $\phi$. (The fact that $\mu$ is $\phi$-invariant ensures that $\Phi_{\mu}$ is well-defined.) We write $\mathcal{B}_{\phi}=$ $\left\{\Phi_{\mu}: \mu \in \mathcal{M}_{\phi}\right\}$. (In the case of geodesic flows, $\mathcal{B}_{\phi}$ is the unit ball for the Gromov-Federer stable norm on homology [10].)

An important invariant describing the complexity of the orbit structure of $\phi$ is the topological entropy $h(\phi)$. For Anosov flows, $h(\phi)>0$ and it is related to the (measure theoretic) entropies $h(\mu)$ of measures in $\mathcal{M}_{\phi}$ by the variational principle:

$$
h(\phi)=\sup _{\mu \in \mathcal{M}_{\phi}} h(\mu) .
$$

There is a unique measure $\mu_{0} \in \mathcal{M}_{\phi}$, called the measure of maximal entropy, for which this supremum is attained. We write $\Phi_{0}=\Phi_{\mu_{0}}$; this winding cycle will play a particularly important role. We note that $\Phi_{0}$ lies in the interior of $\mathcal{B}_{\phi}$.

More generally, we define a (real analytic) function $\mathfrak{h}: \operatorname{int}\left(\mathcal{B}_{\phi}\right) \rightarrow \mathbb{R}$ by $\mathfrak{h}(\rho)=$ $\sup \left\{h_{\phi}(\mu): \Phi_{\mu}=\rho\right\}$. Clearly, $\mathfrak{h}\left(\Phi_{0}\right)=h(\phi)$ and if $\rho \neq \Phi_{0}$ then $\mathfrak{h}(\rho)<h(\phi)$; in particular, $\nabla \mathfrak{h}\left(\Phi_{0}\right)=0$. In fact, it is a well-known result that $\mathfrak{h}$ is strictly concave and that $\mathcal{H}=-\nabla^{2} \mathfrak{h}\left(\Phi_{0}\right)$ is positive definite [2], [9], [15]. In the case $\Phi_{0}=0$, we define the norm $\|\cdot\|$, which appears in Theorem 1 , by $\|\rho\|^{2}=\langle\rho, \mathcal{H} \rho\rangle$. In particular,

$$
\mathfrak{h}(\rho)=h(\phi)-\|\rho\|^{2} / 2+O\left(\|\rho\|^{3}\right) .
$$

We also define $\sigma>0$ by $\sigma^{-2 d}=\operatorname{det} \mathcal{H}$.

We also define a pressure function $\mathfrak{p}: H^{1}(M, \mathbb{R}) \rightarrow \mathbb{R}$ by $\mathfrak{p}([\omega])=P(\omega(\mathcal{X}))$, where the pressure $P(f)$ of a continuous function $f: M \rightarrow \mathbb{R}$ is given by

$$
P(f)=\sup _{\mu \in \mathcal{M}_{\phi}}\left\{h(\mu)+\int f d \mu\right\} \text {. }
$$


If $f$ is Hölder continuous then this supremum is attained for a unique element, which we call the equilibrium state of $f$. When $[\omega]=\xi$, we shall write $\mu_{\xi}$ for the equilibrium state of $\omega(\mathcal{X})$. (If $\xi=0$ then $\mu_{0}$ is the measure of maximal entropy, agreeing with the notation above.)

In the language of convex analysis, $-\mathfrak{h}$ and $\mathfrak{p}$ form a Legendre conjugate pair. More precisely, $\nabla \mathfrak{h}: \mathcal{B}_{\phi} \rightarrow \mathbb{R}^{d}$ and $\nabla \mathfrak{p}: \mathbb{R}^{d} \rightarrow \mathcal{B}_{\phi}$ are inverses and

$$
\mathfrak{h}(\rho)=\mathfrak{p}\left((\nabla \mathfrak{p})^{-1}(\rho)\right)-\left\langle(\nabla \mathfrak{p})^{-1}(\rho), \rho\right\rangle .
$$

We write $\xi(\rho)=(\nabla \mathfrak{p})^{-1}(\rho)$. Then $\nabla^{2} \mathfrak{h}(\rho)=\left(\nabla^{2} \mathfrak{p}(\xi(\rho))\right)^{-1}$ and $\mathfrak{h}(\rho)=h_{\phi}\left(\mu_{\xi(\rho)}\right)$.

\section{Periodic Orbits and Homology}

An Anosov flow has a countable infinity of periodic orbits, whose lengths have no finite accumulation points. If we define $\pi(T)$ to be the number of (prime) periodic orbits of least period at most $T$ then $\pi(T) \sim e^{h(\phi) T} / h(\phi) T$, as $T \rightarrow+\infty$ [8],[9],[11].

In the case of geodesic flow $\phi_{t}: S N \rightarrow S N$, the closed geodesics on $N$ are in one-to-one correspondence with the periodic orbits for $\phi$, with the length of the geodesic equal to the least period of the orbit. (This approach is implicit in the analysis in the appendix.)

The distribution of periodic orbits relative to homology has been extensively studied. To obtain interesting results, we shall suppose that $H_{1}(M, \mathbb{Z})$ is infinite. To each periodic orbit $\gamma$, we can associate a homology class $[\gamma] \in H_{1}(M, \mathbb{Z})$. We shall work modulo any torsion in $H_{1}(M, \mathbb{Z})$ and suppose that, after fixing a basis, that $[\gamma] \in \mathbb{Z}^{k}$, where $k \geq 1$ is the first Betti number of $M$.

As in the introduction, for $\alpha \in H_{1}(N, \mathbb{Z})$, we write $\pi(T, \alpha)=\#\{\gamma: l(\gamma) \leq T,[\gamma]=\alpha\}$. Now, however, we shall allow $\alpha$ to depend on $T$ (in a linear way). To continue to take values in $H_{1}(N, \mathbb{Z})$, we shall define an "integer part" on $H_{1}(M, \mathbb{R})$. Choose a fundamental domain $\mathcal{F}$ for $H_{1}(M, \mathbb{Z})$ as a lattice inside $H_{1}(M, \mathbb{R})$. Then, for $\rho \in H_{1}(M, \mathbb{R})$, define $\lfloor\rho\rfloor \in H_{1}(M, \mathbb{Z})$ by $\rho-\lfloor\rho\rfloor \in \mathcal{F}$.

Proposition $2.1[2],[7],[15]$. Let $\phi_{t}: M \rightarrow M$ be a weak-mixing transitive Anosov flow. If $\rho \in \operatorname{int}\left(\mathcal{B}_{\phi}\right)$ and $\alpha \in H_{1}(M, \mathbb{Z})$ then

$$
\pi(T, \alpha+\lfloor\rho T\rfloor) \sim \frac{\sqrt{\operatorname{det} \nabla^{2} \mathfrak{h}(\rho)}}{(2 \pi)^{k / 2} \mathfrak{h}(\rho)} e^{-\langle\xi(\rho), \alpha\rangle} e^{\langle\xi(\rho), T \rho-\lfloor T \rho\rfloor\rangle} \frac{e^{\mathfrak{h}(\rho) T}}{T^{k / 2+1}}, \quad \text { as } T \rightarrow+\infty,
$$

uniformly for $\rho$ in compact subsets of $\operatorname{int}\left(\mathcal{B}_{\phi}\right)$. In particular, if $\rho \neq \Phi_{0}$ then $\pi(T, \alpha+$ $\lfloor\rho T\rfloor) / \pi(T)$ tends to zero exponentially fast, as $T \rightarrow+\infty$.

Remark. If $\Phi_{0}=0$ then $\phi_{t}: M \rightarrow M$ is automatically weak-mixing [15]. Setting $\rho=0$ in this case recovers the asymptotic formula (0.1).

The following consequence of the Proposition was first stated by Lalley [6]. It may also be deduced from a large deviations result of Kifer [4] and still holds if $\phi$ is not weak-mixing.

Corollary 2.1.1 [4],[6]. For any $\epsilon>0$, we have

$$
\lim _{T \rightarrow+\infty} \frac{1}{\pi(T)} \#\left\{\gamma: l(\gamma) \leq T,\left\|\frac{[\gamma]}{l(\gamma)}-\Phi_{0}\right\|<\epsilon\right\}=1
$$

The next result follows from the uniformity in Proposition 2.1. 
Corollary 2.1.2 [2],[7]. Suppose that $\Phi_{0}=0$. Then, for $\delta>0$ sufficiently small,

$$
\lim _{T \rightarrow+\infty} \sup _{\|\alpha\| \leq \delta T}\left|\frac{T^{k / 2+1} \pi(T, \alpha)}{C(\alpha / T) e^{\mathfrak{h}(\alpha / T) T}}-1\right|=0,
$$

where $C(\rho)=(2 \pi)^{k / 2} \mathfrak{h}(\rho)\left(\operatorname{det} \nabla^{2} \mathfrak{h}(\rho)\right)^{-1 / 2}$.

The following version of the Central Limit Theorem also holds. (We give the statement under the condition $\Phi_{0}=0$, which is the case we need, but the result holds more generally if we replace $[\gamma]$ by $\left.[\gamma]-T \Phi_{0}.\right)$

Corollary 2.1.3 [2],[7]. Suppose that $\Phi_{0}=0$. Then, for a Borel sets $B \subset \mathbb{R}^{k}$ whose boundary has zero measure,

$$
\lim _{T \rightarrow+\infty} \frac{1}{\pi(T)} \#\left\{\gamma: l(\gamma) \leq T, \frac{[\gamma]}{\sqrt{T}} \in B\right\}=\frac{1}{(2 \pi)^{k / 2} \sigma^{k}} \int_{B} e^{-\|x\|^{2} / 2} d x
$$

We end this section be deducing part (ii) of Theorem 1 from Corollary 2.1.1. It follows from this that if $S$ is a sector which includes a neighbourhood of $\Phi_{0}$, then

$$
\lim _{T \rightarrow+\infty} \frac{1}{\pi(T)} \#\left\{\gamma: l(\gamma) \leq T, \frac{[\gamma]}{l(\gamma)} \in S\right\}=1
$$

Hence, because $S$ is a sector,

$$
\lim _{T \rightarrow+\infty} \frac{1}{\pi(T)} \#\{\gamma: l(\gamma) \leq T,[\gamma] \in S\}=1
$$

Rewriting this in terms of homology directions, we have that if $D \subset S^{d-1}$ contains a neighbourhood of $\Phi_{0} /\left\|\Phi_{0}\right\|_{2}$ then

$$
\lim _{T \rightarrow+\infty} \frac{1}{\pi(T)} \#\{\gamma: l(\gamma) \leq T, \theta(\gamma) \in D\}=1
$$

\section{Proof of Theorem 2}

Let $\epsilon>0$ be given and choose $\Delta>0$ such that

$$
\frac{1}{(2 \pi)^{k / 2} \sigma^{k}} \int_{\|x\|>\Delta} e^{-\|x\|^{2} / 2} d x<\epsilon .
$$

Provided $T$ is sufficiently large, $\Delta \sqrt{T} \leq \delta T$, so it follows from Corollary 2.1.2 that

$$
\lim _{T \rightarrow+\infty} \sup _{\|\alpha\| \leq \Delta \sqrt{T}}\left|\frac{T^{k / 2+1} \pi(T, \alpha)}{C(\alpha / T) e^{\mathfrak{h}(\alpha / T) T}}-1\right|=0
$$

or, equivalently, that

$$
\sup _{\|\alpha\| \leq \Delta \sqrt{T}}\left|\frac{T \pi(T, \alpha)}{C(\alpha / T) e^{\mathfrak{h}(\alpha / T) T}}-\frac{1}{T^{k / 2}}\right|=o\left(\frac{1}{T^{k / 2}}\right) .
$$

Since, for $\|\alpha\| \leq \Delta \sqrt{T}$,

(i) $|C(\alpha / T)-C(0)|=O\left(T^{-1 / 2}\right)$, and, using (1.1),

(ii) $\mathfrak{h}(\alpha / T) T=h(\phi)-\|\alpha\|^{2} / 2 T+O\left(T^{-1 / 2}\right)$, 
we may replace this by

$$
\sup _{\|\alpha\| \leq \Delta \sqrt{T}}\left|\frac{h(\phi) T \pi(T, \alpha)}{e^{h(\phi) T}}-\frac{e^{-\|\alpha\|^{2} / 2 T} e^{q(\alpha, T)}}{(2 \pi)^{k / 2} \sigma^{k} T^{k / 2}}\right|=o\left(\frac{1}{T^{k / 2}}\right),
$$

where $e^{q(\alpha, T)} \in\left(e^{-c T^{-1 / 2}}, e^{c T^{-1 / 2}}\right)$, for some $c>0$.

Summing (3.2) over $\alpha \in A$ with $\|\alpha\| \leq \Delta \sqrt{T}$ and using the estimate $\#\{\alpha \in A:\|\alpha\| \leq$ $\Delta \sqrt{T}\}=O\left(T^{k / 2}\right)$ gives

$$
\lim _{T \rightarrow+\infty} \sum_{\substack{\alpha \in A \\\|\alpha\| \leq \Delta \sqrt{T}}}\left(\frac{h(\phi) T \pi(T, \alpha)}{e^{h(\phi) T}}-\frac{e^{-\|\alpha\|^{2} / 2 T} e^{q(\alpha, T)}}{(2 \pi)^{k / 2} \sigma^{k} T^{k / 2}}\right)=0 .
$$

The behaviour of the second term in the summand will follow from Lemma 3.1 below, the proof of which is contained in the next section.

Lemma 3.1. If $A \subset \mathbb{Z}^{k}$ has density $d_{\|\cdot\|}(A)$ (with respect to the norm $\|\cdot\|$ ) then

$$
\lim _{T \rightarrow+\infty} \frac{1}{(2 \pi)^{g} \sigma^{k} T^{k / 2}} \sum_{\substack{\alpha \in A \\\|\alpha\| \leq \Delta \sqrt{T}}} e^{-\|\alpha\|^{2} / 2 T}=d_{\|\cdot\|}(A) .
$$

We may write

$$
\sum_{\substack{\alpha \in A \\\|\alpha\| \leq \Delta \sqrt{T}}} \frac{e^{-\|\alpha\|^{2} / 2 T} e^{q(\alpha, T)}}{(2 \pi)^{k / 2} \sigma^{k} T^{k / 2}}=\sum_{\substack{\alpha \in A \\\|\alpha\| \leq \Delta \sqrt{T}}} \frac{e^{-\|\alpha\|^{2} / 2 T}}{(2 \pi)^{k / 2} \sigma^{k} T^{k / 2}}+\sum_{\substack{\alpha \in A \\\|\alpha\| \leq \Delta \sqrt{T}}} \frac{e^{-\|\alpha\|^{2} / 2 T}\left(e^{q(\alpha, T)}-1\right)}{(2 \pi)^{k / 2} \sigma^{k} T^{k / 2}}
$$

and, since $\left|e^{q(\alpha, T)}-1\right|$ is uniformly bounded by $e^{c T^{-1 / 2}}-1$, it follows from Lemma 3.1 that

$$
\lim _{T \rightarrow+\infty} \sum_{\substack{\alpha \in A \\\|\alpha\| \leq \Delta \sqrt{T}}} \frac{e^{-\|\alpha\|^{2} / 2 T} e^{q(\alpha, T)}}{(2 \pi)^{k / 2} \sigma^{k} T^{k / 2}}=d_{\|\cdot\|}(A) .
$$

We are now ready to complete the proof of Theorem 2 . Given that $\pi(T) \sim e^{h(\phi) T} / h(\phi) T$, as $T \rightarrow+\infty, \pi(T, A) / \pi(T)$ and $h(\phi) T \pi(T, A) / e^{h(\phi) T}$ have the same limit. We have

$$
\frac{h(\phi) T \pi(T, A)}{e^{h(\phi) T}}=\sum_{\substack{\alpha \in A \\\|\alpha\| \leq \Delta \sqrt{T}}} \frac{h(\phi) T \pi(T, \alpha)}{e^{h(\phi) T}}+\sum_{\substack{\alpha \in A \\\|\alpha\|>\Delta \sqrt{T}}} \frac{h(\phi) T \pi(T, \alpha)}{e^{h(\phi) T}}
$$

and, by Corollary 2.1.3,

$$
\begin{aligned}
0 \leq \limsup _{T \rightarrow+\infty} \sum_{\substack{\alpha \in A \\
\|\alpha\|>\Delta \sqrt{T}}} \frac{h(\phi) T \pi(T, \alpha)}{e^{h(\phi) T}} & \leq \lim _{T \rightarrow+\infty} \sum_{\|\alpha\|>\Delta \sqrt{T}} \frac{h(\phi) T \pi(T, \alpha)}{e^{h(\phi) T}} \\
& =\frac{1}{(2 \pi)^{k / 2} \sigma^{k}} \int_{\|x\|>\Delta} e^{-\|x\|^{2} / 2} d x .
\end{aligned}
$$


Hence

$$
\liminf _{T \rightarrow+\infty} \frac{h(\phi) T \pi(T, A)}{e^{h(\phi) T}} \geq \lim _{T \rightarrow+\infty} \sum_{\substack{\alpha \in A \\\|\alpha\| \leq \Delta \sqrt{T}}} \frac{h(\phi) T \pi(T, \alpha)}{e^{h(\phi) T}}=d_{\|\cdot\|}(A)
$$

and, using (3.1),

$$
\begin{aligned}
\limsup _{T \rightarrow+\infty} \frac{h(\phi) T \pi(T, A)}{e^{h(\phi) T}} & \leq \lim _{T \rightarrow+\infty} \sum_{\substack{\alpha \in A \\
\|\alpha\| \leq \Delta \sqrt{T}}} \frac{h(\phi) T \pi(T, \alpha)}{e^{h(\phi) T}}+\limsup _{T \rightarrow+\infty} \sum_{\substack{\alpha \in A \\
\|\alpha\|>\Delta \sqrt{T}}} \frac{h(\phi) T \pi(T, \alpha)}{e^{h(\phi) T}} \\
& <d_{\|\cdot\|}(A)+\epsilon .
\end{aligned}
$$

Since $\epsilon>0$ is arbitrary, Theorem 2 follows.

\section{Proof of Lemma 3.1}

Lemma 3.1 may be deduced from the following more general result.

Lemma 4.1. Let $\|\cdot\|$ be an arbitrary norm on $\mathbb{R}^{k}$ and let $f: \mathbb{R}^{k} \rightarrow \mathbb{R}$ be a continuous integrable function such that $f(t)$ depends only on $\|t\|$. Suppose also that $|f| \leq F$ where $F: \mathbb{R}^{k} \rightarrow \mathbb{R}^{+}$is an integrable function depending only on $\|t\|$, such that $F(\|t\|)$ is decreasing in $\|t\|$. If $A \subset \mathbb{Z}^{k}$ has density $d_{\|\cdot\|}(A)$ (with respect to $\|\cdot\|$ ) then

$$
\lim _{x \rightarrow+\infty} \sum_{\alpha \in A} x^{-k} f(\alpha / x)=d_{\|\cdot\|}(A) \int_{\mathbb{R}^{k}} f(t) d t .
$$

Remark. The condition that $f$ is bounded by an integrable function decreasing in $\|t\|$ is stronger than is necessary. However some control is needed on the behaviour of $f$ as $\|t\| \rightarrow+\infty$, as there exist continuous integrable functions $f$ for which the desired limit does not exist.

Proof. We first show that, for any $\epsilon>0$, we can find $R>0$ such that, for all $x>1$,

$$
\sum_{\substack{\alpha \in \mathbb{Z}^{k} \\\|\alpha\|>x R}} x^{-k}|f(\alpha / x)|<\epsilon
$$

For any $\alpha \in \mathbb{Z}^{k}, \alpha \neq 0$, define

$$
\alpha^{\prime}=\left(\|\alpha\|_{2}-1 / 4\right)\|\alpha\|^{-1} \alpha \in \mathbb{R}^{k},
$$

where $\|\cdot\|_{2}$ is the usual 2-norm on $\mathbb{R}^{k}$. Because any two norms on $\mathbb{R}^{k}$ are equivalent, we can find constants $c_{1}, c_{2}>0$ such that $c_{1}\|t\|_{2} \leq\|t\| \leq c_{2}\|t\|_{2}$, for any $t \in \mathbb{R}^{k}$. By applying this to $t=\alpha^{\prime}-\alpha$, we see that for any $\alpha \neq 0,\left\|\alpha^{\prime}\right\| \leq\|\alpha\|-c_{1} / 4$.

Now let $r=c_{1} / 4 c_{2}$, and consider $B_{2}\left(\alpha^{\prime}, r\right)$, the open ball centre $\alpha^{\prime}$ and radius $r$ in the 2 -norm. This ball has a volume $v$ which is independent of $\alpha$, and, for different values of 
$\alpha$, these balls are disjoint. Note also that if $t \in B_{2}\left(\alpha^{\prime}, r\right)$ then $\left\|t-\alpha^{\prime}\right\|<c_{1} / 4$ and so $\|t\|<\|\alpha\|$.

Similarly, if $x \geq 1$ then for any $t \in B_{2}\left(\alpha^{\prime} / x, r / x\right)$ we have $\|t\|<\|\alpha / x\|$, and so $F(t) \geq$ $F(\alpha / x)$. Hence

$$
\int_{B_{2}\left(\alpha^{\prime} / x, r / x\right)} F(t) d t \geq v x^{-k} F(\alpha / x) \geq v x^{-k}|f(\alpha / x)|
$$

Summing over $\|\alpha\| \geq x R$ and using the fact that the balls $B_{2}\left(\alpha^{\prime} / x, r / x\right)$ are disjoint, we obtain

$$
\sum_{\substack{\alpha \in \mathbb{Z}^{k} \\\|\alpha\|>x R}} x^{-k}|f(\alpha / x)| \leq \frac{1}{v} \sum_{\substack{\alpha \in \mathbb{Z}^{k} \\\|\alpha\|>x R}} \int_{B_{2}\left(\alpha^{\prime} / x, r / x\right)} F(t) d t \leq \frac{1}{v} \int_{\|t\|>R-1} F(t) d t
$$

However, $F$ is integrable, so this last integral can be made as small as desired by choosing $R$ sufficiently large. This completes the preliminary part of the proof.

It follows from this that it is sufficient to prove that, for large $R$,

$$
\lim _{x \rightarrow+\infty} \sum_{\substack{\alpha \in A \\\|\alpha\| \leq x R}} x^{-k} f(\alpha / x)=d_{\|\cdot\|}(A) \int_{\|t\| \leq R} f(t) d t
$$

We may at this point assume that $f$ is strictly positive. (If not, we can write $f$ in terms of its positive and negative parts, $f=f_{+}-f_{-}$, and consider $f_{+}+1$ and $f_{-}+1$.)

The case $A=\mathbb{Z}^{k}$ is straightforward from the Riemann definition of integration. We wish to extend this result to a general set $A$ for which $d_{\|\cdot\|}(A)$ exists. For a large integer $n$, define the sets

$$
\begin{aligned}
S_{1} & :=\left\{t \in \mathbb{R}^{k}:\|t\| \leq R n^{-1}\right\} \\
S_{m} & :=\left\{t \in \mathbb{R}^{k}: R(m-1) n^{-1}<\|t\| \leq R m n^{-1}\right\}, \quad 2 \leq m \leq n
\end{aligned}
$$

so that $\{t:\|t\| \leq R\}$ is their disjoint union. We claim that for any $m$,

$$
P_{m}(x, A):=\frac{\#\left\{\alpha \in A: x^{-1} \alpha \in S_{m}\right\}}{\#\left\{\alpha \in \mathbb{Z}^{k}: x^{-1} \alpha \in S_{m}\right\}} \rightarrow d_{\|\cdot\|}(A), \text { as } x \rightarrow+\infty .
$$

For $m=1$ this is immediate from the definition of the density. For $m>1$, we have

$$
P_{m}(x, A)=\frac{\#\left\{\alpha \in A: x^{-1}\|\alpha\| \leq R m n^{-1}\right\}-\#\left\{\alpha \in A: x^{-1}\|\alpha\| \leq R(m-1) n^{-1}\right\}}{\#\left\{\alpha \in \mathbb{Z}^{k}: x^{-1}\|\alpha\| \leq R m n^{-1}\right\}-\#\left\{\alpha \in \mathbb{Z}^{k}: x^{-1}\|\alpha\| \leq R(m-1) n^{-1}\right\}} .
$$

Now, by the definition of $d_{\|\cdot\|}$, we have

$$
\lim _{x \rightarrow+\infty} \frac{\#\left\{\alpha \in A: x^{-1}\|\alpha\| \leq R m n^{-1}\right\}}{\#\left\{\alpha \in \mathbb{Z}^{k}: x^{-1}\|\alpha\| \leq R m n^{-1}\right\}}=d_{\|\cdot\|}(A) .
$$


Also

$$
\lim _{x \rightarrow+\infty} x^{-k} \#\left\{\alpha \in \mathbb{Z}^{k}: x^{-1}\|\alpha\| \leq R m n^{-1}\right\}=\operatorname{Vol}\left\{t:\|t\| \leq R m n^{-1}\right\},
$$

which follows from the case $A=\mathbb{Z}^{k}$ by letting $f$ approximate the indicator function of $\left\{t:\|t\| \leq R m n^{-1}\right\}$. Hence, as $x \rightarrow+\infty, P_{m}(x, A)$ converges to

$$
\begin{aligned}
d_{\|\cdot\|}(A) & \left(\frac{\operatorname{Vol}\left\{t:\|t\| \leq R m n^{-1}\right\}}{\operatorname{Vol}\left\{t:\|t\| \leq R m n^{-1}\right\}-\operatorname{Vol}\left\{t:\|t\| \leq R(m-1) n^{-1}\right\}}\right) \\
- & d_{\|\cdot\|}(A)\left(\frac{\operatorname{Vol}\left\{t:\|t\| \leq R(m-1) n^{-1}\right\}}{\operatorname{Vol}\left\{t:\|t\| \leq R m n^{-1}\right\}-\operatorname{Vol}\left\{t:\|t\| \leq R(m-1) n^{-1}\right\}}\right)=d_{\|\cdot\|}(A) .
\end{aligned}
$$

We now use the fact that $f(t)$ depends only on $\|t\|$. Together with the assumption that $f$ is positive and (uniformly) continuous on $\|t\|<R$, this implies that for any $\epsilon>0$, we can choose $n$ sufficiently large that

$$
\max _{t \in S_{m}} f(t)<(1+\epsilon) \min _{t \in S_{m}} f(t)
$$

simultaneously for all $1 \leq m \leq n$. In addition, for each such $n$, we know that if $x$ is sufficiently large then for all $1 \leq m \leq n, d_{\|\cdot\|}(A)-\epsilon<P_{m}(x, A)<d_{\|\cdot\|}(A)+\epsilon$. Then

$$
\begin{aligned}
\sum_{\substack{\alpha \in A \\
x^{-1} \alpha \in S_{m}}} x^{-k} f(\alpha / x) & \geq \sum_{\substack{\alpha \in A \\
x^{-1} \alpha \in S_{m}}} x^{-k} \min _{t \in S_{m}} f(t) \geq\left(d_{\|\cdot\|}(A)-\epsilon\right) \sum_{\substack{\alpha \in \mathbb{Z}^{k} \\
x^{-1} \alpha \in S_{m}}} x^{-k} \min _{t \in S_{m}} f(t) \\
& \geq \frac{d_{\|\cdot\|}(A)-\epsilon}{1+\epsilon} \sum_{\substack{\alpha \in \mathbb{Z}^{k} \\
x^{-1} \alpha \in S_{m}}} x^{-k} f(\alpha / x),
\end{aligned}
$$

so, by summing over $m$,

$$
\sum_{\substack{\alpha \in A \\\|\alpha\| \leq x R}} x^{-k} f(\alpha / x) \geq \frac{d_{\|\cdot\|}(A)-\epsilon}{1+\epsilon} \sum_{\substack{\alpha \in \mathbb{Z}^{k} \\\|\alpha\| \leq x R}} x^{-k} f(\alpha / x) .
$$

Taking $x \rightarrow+\infty$ and using the result for the case $A=\mathbb{Z}^{k}$ gives

$$
\liminf _{x \rightarrow+\infty} \sum_{\substack{\alpha \in A \\\|\alpha\| \leq x R}} x^{-k} f(\alpha / x) \geq \frac{d_{\|\cdot\|}(A)-\epsilon}{1+\epsilon} \int_{\|t\| \leq R} f(t) d t .
$$

A similar argument shows

$$
\limsup _{x \rightarrow+\infty} \sum_{\substack{\alpha \in A \\\|\alpha\| \leq x R}} x^{-k} f(\alpha / x) \leq\left(d_{\|\cdot\|}(A)+\epsilon\right)(1+\epsilon) \int_{\|t\| \leq R} f(t) d t .
$$


Since $\epsilon>0$ is arbitrary, this completes the proof.

Proof of Lemma 3.1. Since

$$
\int_{\mathbb{R}^{k}} e^{-\langle t, \mathcal{H} t\rangle / 2} d t=\frac{(2 \pi)^{k / 2}}{\sqrt{\operatorname{det} \mathcal{H}}}
$$

applying Lemma 4.1 with $f(t)=e^{-\|t\|^{2} / 2}$ and $T=x^{2}$ gives immediately that

$$
\lim _{T \rightarrow+\infty} \frac{1}{(2 \pi)^{k / 2} \sigma^{k} T^{k / 2}} \sum_{\alpha \in A} e^{-\|\alpha\|^{2} / 2 T}=d_{\|\cdot\|}(A) .
$$

Finally, increasing $\Delta$ if necessary, we may suppose that $\Delta \geq R$ and so, by inequality (4.1),

$$
\frac{1}{T^{k / 2}} \sum_{\substack{\alpha \in A \\\|\alpha\|>\Delta \sqrt{T}}} e^{-\|\alpha\|^{2} / 2 T}<\epsilon .
$$

This completes the proof.

\section{REFERENCES}

1. N. Anantharaman, Precise counting results for closed orbits of Anosov flows, Ann. Sci. École Norm. Sup. 33 (2000), 33-56.

2. M. Babillot and F. Ledrappier, Lalley's theorem on periodic orbits of hyperbolic flows, Ergodic Theory Dyn. Syst. 18 (1998), 17-39.

3. D. Fried, The geometry of cross sections to flows, Topology 21 (1982), 353-371.

4. Y. Kifer, Large deviations, averaging and periodic orbits of dynamical systems, Comm. Math. Phys. 162 (1994), 33-46.

5. M. Kotani, A note on asymptotic expansions for closed geodesics in homology classes, Math. Ann. 320 (2001), 507-529.

6. S. Lalley, Distribution of periodic orbits of symbolic and Axiom A flows, Adv. Appl. Math. 8 (1987), 154-193.

7. S. Lalley, Closed geodesics in homology classes on surfaces of variable negative curvature, Duke Math. J. 58 (1989), 795-821.

8. G. Margulis, On some applications of ergodic theory to the study of manifolds on negative curvature, Fun. Anal. Appl. 3 (1969), 89-90.

9. G. Margulis, On some aspects of the theory of Anosov systems. With a survey by Richard Sharp: Periodic orbits of hyperbolic flows, Springer Monographs in Mathematics, Springer-Verlag, Berlin, 2004.

10. D. Massart, Stable norms of surfaces: local structure of the unit ball of rational directions, Geom. Funct. Anal. 7 (1997), 996-1010.

11. W. Parry and M. Pollicott, Zeta functions and the periodic orbit structure of hyperbolic dynamics, Astérisque 187-88 (1990), 1-268.

12. Y. Petridis and M . Risager, Equidistribution of geodesics on homology classes and analogues for free groups, preprint (2006).

13. M. Pollicott and R. Sharp, Asymptotic expansions for closed orbits in homology classes, Geom. Dedicata 87 (2001), 123-160.

14. S. Schwartzman, Asymptotic cycles, Ann. of Math. 66 (1957), 270-284.

15. R. Sharp, Closed orbits in homology classes for Anosov flows, Ergodic Theory Dyn. Syst. 13 (1993), 387-408.

16. R. Sharp, A local limit theorem for closed geodesics and homology, Trans. Amer. Math. Soc. 356 (2004), 4897-4908.

Department of Mathematics, University of Manchester, Oxford Road, Manchester M13 9PL, U.K. 